\title{
Networked Monitoring and Control System of Tyre Building Machine Based on Virtual Instrument
}

\author{
Yang Shifeng ${ }^{a}$, Yu Tao ${ }^{b}$
}

College of Electronic Information and Automation, Tianjin University of Science and Technology, Tianjin, 300222, China

ayangsf@tust.edu.cn, bteresayu0603@126.com

Keywords: LabVIEW, networked, TCP/IP, monitoring and control system

\begin{abstract}
Networked monitoring and control system of tyre building machine is a LAN management system characterized by possessing servers and multiple clients, which are developed by LabVIEW, the virtual instrument development platform and combining with network information technologies such as TCP/IP and OPC. It uses customized communication protocol based on TCP/IP protocol, which makes the data management and interactions between server and clients faster and more conveniently. Using C/S model as the basis of the system construction, it ultimately achieves varied functions including alarms warning, rights management, invoking the formula information of multiple clients arbitrarily and summarizing the yield. The system designed brings great convenience to the management of multiple clients, which eventually makes the process of tyre manufacture more automatic and intelligent.
\end{abstract}

\section{Introduction}

Tyre manufacturing is an important part of automobile manufacturing. Since the inception of the new century, tyre enterprises got an unprecedented opportunity with the rapid development of automobile manufacturing. Improving production efficiency and automation degree of TBM (Tyre Building Machine) is crucial to the tyre manufacturing [1].

In recent years, with the rapid development of computer and network technology, people's life and way of thinking is being changed by network rapidly. At the same time, modern industry has achieved a high degree of automation through the network, which can realize real-time output information and alarm information sharing function. With the continuous improvement of network speed and stability, the use of existing Internet network to set up the network measurement and control system is the future development direction of virtual instrument technology.

In this paper, using the development of virtual instrument on the data processing, generality and extensibility, combining with the modern network, TBM network monitoring detection and control system is built based on virtual instrument to meet increasing industrial production requirements.

\section{System Structure and Working Principle}

Monitoring system consists of TBM, PLC and IPCs. Sensors convert the parameters that the monitoring and control system needed into standard electrical signals which are then transmitted to PLC through the input module. At last, PLC sends the processed data to the upper computer by OPC server and the monitoring and control system is responsible for the terminal data processing and displaying [2]. The server is used to receive and store alarm information, output information and user information from other client IPCs. At the same time, some data in the server can be called by any client IPCs. The structure of this system is shown in figure 1. 


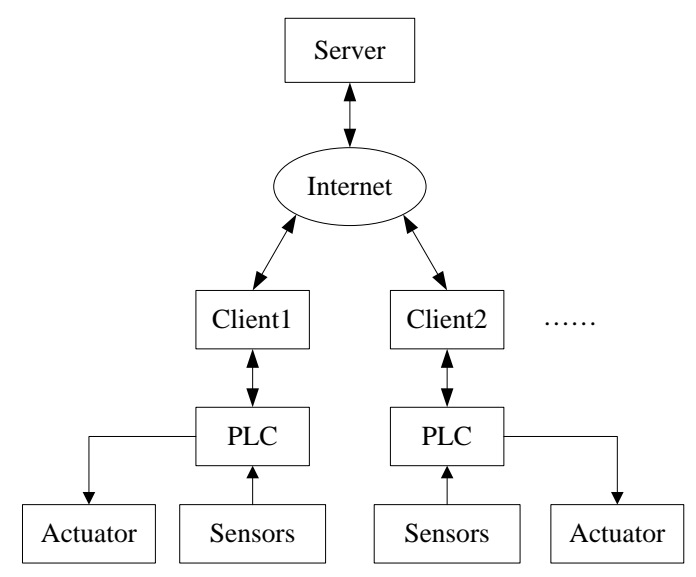

Fig.1 Structure chart of the system

\section{Network Model of Networked Monitoring and Control System}

C/S Model. The C/S (Client/Server) model uses server as data storage and exchange platform and operate data by clients. It has the advantages of high degree of specialization, flexibility of development and fast operation speed. It applies to almost any platform and has a variety of development techniques. But it also has some disadvantages such as large maintenance costs and weak adaptability.

B/S Model. B/S (Browser/Server) model is a change and improvement on C/S under the development of Internet. Client can visit the monitoring and control website and do something as simple as reading data using browser. It has many advantages such as easy upgrade, easy maintenance and high flexibility. But it is restricted by problems of low agility and few functions.

Comparison of $\mathbf{C} / \mathbf{S}$ and B/S Model. Although B/S has advantages in difficulties of development and transplantation, it can only realize browsing, query, data input and other simple functions and cannot meet the requirements of controlling the production process by IPC. Therefore, C/S mode network is used in this system.

\section{Network Communication Mode of Virtual Instruments}

TCP/IP. TCP/IP as the core of network communication protocols, it supports the data communication between different platforms and applications, and has the ability for failure recovery and error handling. At the same time, it also occupies a smaller cyber source which ensure the smooth of network. TCP/IP protocol cluster contains the link layer, network layer, transport layer and application layer.[3].

DataSocket. DataSocket is a network communication technology for measurement developed by NI (National Instrument company). It contains three major sections, DataSocket Server Manager, DataSocket Server and DataSocket API. DataSocket Server is a stand-alone program, providing places for sending and receiving data. DataSocket Server Manager is used to configure DataSocket Server. DataSocket APIs provide some sample application interface for programmers to read and write data to DataSocket Server. Programmers won't need to write different code for different format and communication protocol. So it is easier and more efficient for programmer, and it could be used by programmers who do not understand the underlying operating process [4].

Comparison. Although DataSocket packs TCP/IP protocol deeply and makes completing monitoring project more easily, it also has some disadvantages which cannot overcome, such as its transmission performance would significantly reduce if the transfer file larger than $1 \mathrm{M}$ and it has many technical problems for building the remote monitoring and control system. In addition, the client for monitoring and control the remote device is limited by the access permission and the conditions of others. Therefore, self-defined protocol based on TCP/IP protocol cluster is used to realize communication between clients and server in this article. 


\section{The Implementation of Networked Monitoring and Control System of TBM}

Software writing of server and client is the key designing parameter in this system. The server is used to receive and store production and error information, store all recipes of TBMs, and manage users. The client is used to receive data from PLC, monitor and control the process through the program, process and store data and communicate with the server.

LabVIEW is selected as the development platform of server and client. LabVIEW is a graphical programs language developed by NI. It has the characters of good openness, high code reuse rate, low price and convenient connection with Internet and peripherals, which can reduce development costs and time of the monitoring and control system [5].

Build Server. A server is built in this monitoring and control system to manage output data uniformly. The functions of server are described below.

Login. Users are graded according to their name, and they can operate the server depending on their different permission. This ensures safety of the system data.

Clients Management. The user information database is stored in server. Clients need to send username and password to server to verify whether it has permission when start up or switch user.

Store and View Output Data of All Clients. Clients send their real-time output data to server, and server will save them to database. Meanwhile, operators with permission can view output data of all clients in human-machine interface.

Alarm. All alarms will be displayed in server.

Recipe Management. Every client can call any recipe of other clients'. It changes the method of entering recipes manually one by one, and makes operation more convenient.

Communication Process. The system used TCP functions that the LabVIEW function library built-in to realize communication based on TCP/IP. The program flow chart of communication is shown in Figure 2. TCP/IP protocol family should be installed correctly in the computer. And make sure all source ports and target ports needed are opened.

The server should start firstly and then the server starts listening to the communication port and waiting for connection requests. Clients can input the IP address of the server and communication port through the administration interface to realize the communication with the server.

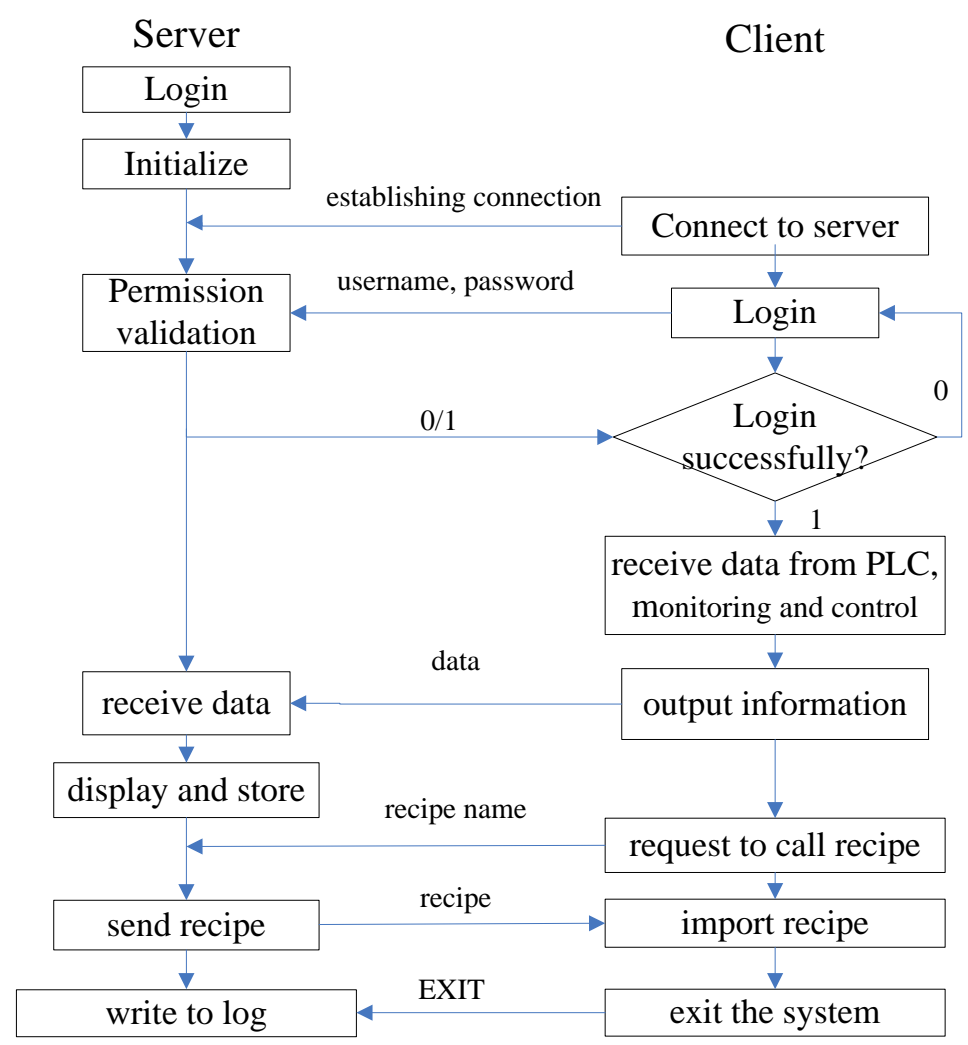

Fig.2 Program flow chart of communication 
Customized Application Layer Communication Protocol. Customized application layer communication protocols of TCP/IP can make the communication more flexible and more applicable to actual projects [6]. The data packet in this system is consist of three parts: Command, Size and Data. The size of data is given by Size. And Commands are listed by Table 1.

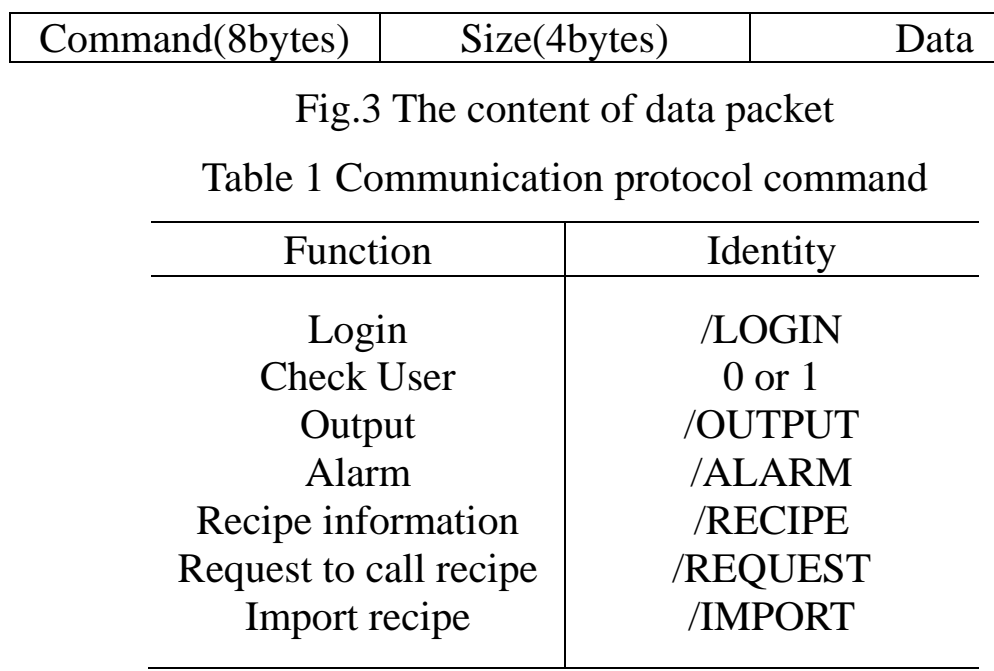

\section{Summary}

According to the actual need of production process, TBM network monitoring and control system combines many TBMs to create a network by the development platform of LabVIEW. By comparisons when constructing the networked system, we finally used C/S model and TCP/IP communication protocol, and customized the application layer based on the transport layer to facilitate the development of program and manage data. This system has been applied in many tyre manufacturers, the practice shows that the networked monitoring and control system of TBM largely reduces human intervention, increases safety and realizes further automation of the tyre production process.

\section{References}

[1] HuJin. Tyre molding machine control system based on Control Logix[D]. Qingdao. College of Automation Engineering of Qiingdao University.2007

[2] Xavier Desforges, Abdallah Habbadi, Laurent Geneste et al. Distributed machining control and monitoring using smart sensors/actuators[J]. Journal of Intelligent Manufacturing, 2004, 15(1).

[3] Danilo Garbi Zutin, Michael E Auer,J.F, Bocanegra, Martins,Juan Antonio Ortega,Andreas Pester. TCP/IP Communication between Server and Client in Multi User Remote Lab Applications[J]. International Journal of Online Engineering, 2008,43:.

[4] Xiaojing Huo, Jinguo Zhang, Wendi Wang,Wei Guo, Jianguo Li. Remote Data Acquisition System at The Dairy Farm Based on DataSocket Technology[J]. Energy Procedia, 2011,13:.

[5] Zhongyuan Wang, Yongheng Shang,Jiarui Liu,Xidong Wu. A LabVIEW based automatic test system for sieving chips[J]. Measurement, 2013,461:.

[6] Celina P Leão,Filomena O Soares, José Machado,Eurico Seabra, Helena Rodrigues. Design and Development of an Industrial Network Laboratory[J]. International Journal of Emerging Technologies in Learning, 2011,6S1:. 